\section{BD-07 GENE TRANSCRIPTS EXPRESSED IN PERIPHERAL BLOOD OF PATIENTS WITH LUPUS NEPHRITIS IDENTIFY NOVEL MECHANISMS AND THERAPEUTIC TARGETS}

Mikhail Olferiev, Kyriakos A Kirou, David R Fernandez, Kerri Merritt, Dina Greenman, Mary K Crow*. Mary Kirkland Center for Lupus Research, Hospital for Special Surgery, New York, NY, USA

\subsection{6/lupus-2018-Ism.31}

Background Lupus nephritis affects more than one-half of patients with SLE and is the most common serious manifestation of the disease. Lupus nephritis is more common in Hispanic and African-American patients than in those of European ancestry, and class III and IV nephritis progresses to end-stage renal disease in 10\%-15\% of patients within 15 years of diagnosis. Identification of markers and mechanisms of lupus nephritis could provide new approaches to predict and treat disease.

Methods To identify blood cell transcriptome biomarkers that differentiate renal and non-renal disease we performed RNA sequencing on peripheral blood samples from 15 patients with lupus nephritis and 14 patients with non-nephritis manifestations of SLE (samples represented each patient during flaring and quiescent disease states) and from 5 healthy donors. To relate gene expression to activity of nephritis, 216 longitudinal samples from 30 patients with lupus nephritis covering a median time frame of 28 months were analyzed using the Illumina HT-V4 Bead array. Serum albumin levels were documented at the time of each visit.

Results Principal component analysis of RNA sequencing data clearly differentiated SLE patients with nephritis from those without nephritis, and linear models for microarray (limma) analysis identified 153 gene transcripts differentially expressed between the two patient groups (fold change $>1.5 ; \mathrm{p}<0.05$ ). $\mathrm{U} 1$ and U3 RNA transcripts were increased in lupus nephritis samples, and the most highly expressed transcript based on fold change was TREML4, encoding a protein previously identified as amplifying TLR7 signaling and promoting type I interferon production. Analysis of longitudinal microarray data in relation to serum albumin identified 120 transcripts. Those most significantly correlated with lupus nephritis activity were pituitary tumor-transforming gene 1 (PTTG1), recently identified as polymorphic and associated with SLE, uridine cytosine kinase 2 (UCK2), thioredoxin (TXN), and RNASE2. Expression of PTTG1 fluctuated over time, with elevated levels preceding the time of peak renal disease activity.

Conclusions Spliceosome-associated RNAs and TREML4, a TLR7-associated gene product, may represent biomarkers of lupus nephritis, and PTTG1, the product of a lupus-associated gene reported to be involved in epithelial-mesenchymal transition, may be a novel therapeutic target associated with active nephritis. These studies provide a rich data set stimulating new understanding of mechanisms contributing to lupus nephritis.

Acknowledgements This work was supported by the Lupus Research Alliance, Pfizer-Centers for Therapeutic Innovation, and the Emerald Foundation.

\section{BD-08 A NOVEL APPROACH TO ANALYZE SINGLE CELL RNA- SEQ DATA FROM LUPUS NEPHRITIS SAMPLES}

Brian J Kegerreis, Amrie C Grammer, Peter E Lipsky*. RILITE Research Institute, Charlottesville, VA, USA

\subsection{6/lupus-2018-Ism.32}

Background Single-cell RNA-Seq (scRNA-seq) has the potential to increase our understanding of cell populations in lupus. Recently, kidney scRNA-Seq data from lupus nephritis (LN) patients has provided the opportunity to determine the heterogeneity of cells within the affected kidney. However, since individual cells were not identified phenotypically, it is necessary to identify populations computationally. The unique technical challenges of scRNA-Seq data make it difficult to approach this analysis with conventional unsupervised bioinformatics techniques. The implementation of natural language processing (NLP) -inspired techniques, however, makes it possible to identify meaningful clusters of cells without prior knowledge of the cell types present in the sample.

Methods We have developed a recursive, unsupervised, heuristic technique (StarShipTM) to dynamically perform top-down, divisive clustering on scRNA-Seq data. First, the cells are mapped onto an $\mathrm{n}$-dimensional unit sphere, where $\mathrm{n}$ is the number of available genes. The angles between all cells are used to construct a cosine distance metric: $1-\cos (\theta)$. The cosine distance is used to carry out k-means or k-medoids clustering, with k set to 2 for each iteration. At each split of the data, the algorithm evaluates whether it has sorted the remaining cells into meaningful populations and stops making splits when a user-defined criterion is met. Once all clusters are finalized, a Mann-Whitney $U$ test determines genes that distinguish clusters or groups of clusters from other cells. This algorithm was validated using publicly available peripheral blood mononuclear cell (PBMC) scRNA-Seq data from 10X Genomics and tested in scRNA-Seq data from LN patients from the NIAMS AMP RA/SLE initiative. Adjusted Rand Index (ARI) was used to compare generated partitions to known cell types in the PBMC data.

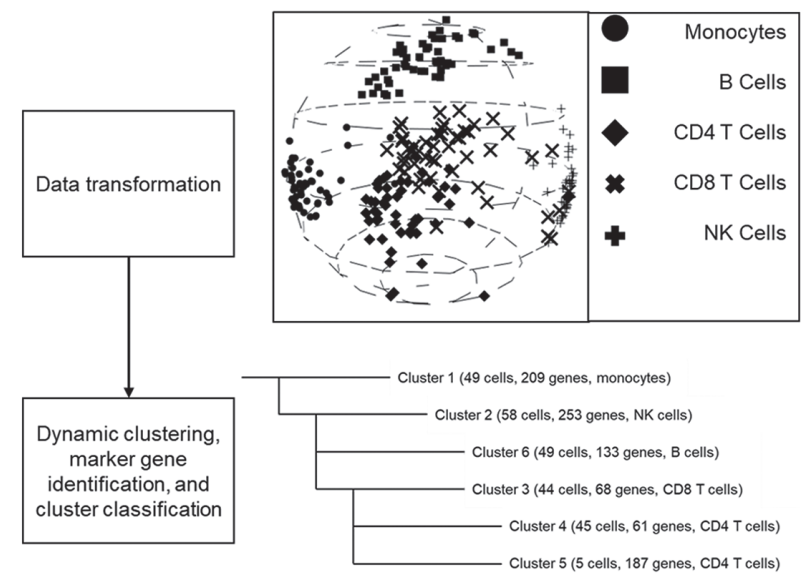

Abstract BD-08 Figure 1 
Results StarShipTM was used to classify 250 PBMC (50 each of CD14 monocytes, CD19 B cells, CD4 helper T cells, CD8 $\mathrm{T}$ cells, and CD56 NK cells). Using dynamic spherical kmeans, 6 clusters were generated that closely corresponded to the known cell types (figure 1). For comparison, hierarchical clustering and one-off spherical k-means with $\mathrm{k}$ set to 5 were carried out. Hierarchical clustering had an ARI of 0.45 , oneoff spherical k-means had an ARI of 0.89 , and dynamic spherical k-means had an ARI of 0.86 .

Conclusions This method can effectively partition unknown cells from scRNA-Seq data sets into biologically relevant clusters without prior knowledge of the number of cell types present. The similarity between the performance of the StarShipTM algorithm and one-off k-means, which does incorporate this prior knowledge, highlights the value of this dynamic technique. A full analysis of the AMP LN data is forthcoming. Acknowledgments Research supported by the RILITE Foundation.

\section{BD-09 PRELIMINARY REPORT: RULE-BASED ALGORITHMS USING SYSTEMIC LUPUS INTERNATIONAL COLLABORATING CLINICS (SLICC) CLASSIFICATION CRITERIA TO IDENTIFY PATIENTS WITH SYSTEMIC LUPUS ERYTHEMATOSUS (SLE) FROM ELECTRONIC HEALTH RECORD (EHR) DATA}

${ }^{1,2}$ Anika S Ghosh, ${ }^{1,2}$ Theresa L Walunas, ${ }^{1,2}$ Kathryn L Jackson, ${ }^{1}$ Anh $\mathrm{H}$ Chung, ${ }^{1}$ Daniel L Erickson, ${ }^{1}$ Karen Mancera-Cuevas, ${ }^{1,2}$ Abel N Kho, ${ }^{1}$ Rosalind Ramsey-Goldman*. ${ }^{1}$ Northwestern University Feinberg School of Medicine, Chicago, IL, USA; ${ }^{2}$ Center for Health Information Partnerships, Chicago, IL, USA

\subsection{6/lupus-2018-Ism.33}

Background SLE is difficult to diagnose given diverse manifestations that occur over time and across care sites. Electronic health records (EHR) are now used in a majority of health care settings throughout the country, and present a rich source of information about patients which can be mined for earlier diagnosis identification, to improve quality of care, or enable clinical studies. To identify SLE patients in EHR data, we developed a rules-based algorithm based on the SLICC classification criteria and compared against a gold standard SLE patient registry data set.

Methods We identified 513 patients in the Chicago Lupus Database (CLD) fulfilling 4 or more of the ACR classification criteria for SLE who also had records in the Northwestern Medicine Electronic Data Warehouse (NMEDW). ICD-9/10 codes were used to identify clinical SLICC SLE classification criteria items. Laboratory results were identified using lab test names in combination with threshold numeric values in order to determine whether patients met the SLICC lab test classification criteria requirements.

Results As shown in table 1, of 513 patients with SLE in the CLD, we detected the following SLICC classification criteria, in the NMEDW: clinical- chronic cutaneous 97\%; acute cutaneous 98\%; renal 65\%; serositis 52\%; arthritis 34\%; neuro $29 \%$; ulcers $16 \%$; alopecia $1 \%$; and labs- dsDNA $89 \%$; hemolytic anemia $80 \%$; complement $74 \%$; leukopenia/lymphopenia 73\%; APL 64\%; ANA 52\%; thrombocytopenia 22\%; Coombs 17\%; Sm 0\%.

Of 513 patients with SLE in the CLD based on ACR criteria, 513 had at least 1 clinical criteria, 466 had at least 1 immunologic criteria, and 471 had 4 or more criteria. Using EHR data from the NMEDW, and rules for the SLICC classification criteria that were based on ICD9/10 codes and labs and required identification of at least one clinical and one immunological criteria, we categorized 450/513 (88\%) patients as having definite lupus.

\begin{tabular}{|c|c|c|c|}
\hline SLICC Criteria & $\begin{array}{l}\text { Identified in } \\
\text { CLD } \\
\text { (N) }\end{array}$ & $\begin{array}{l}\text { Identified in } \\
\text { NMEDW (N) }\end{array}$ & $\begin{array}{l}\text { Identified in CLD and } \\
\text { NMEDW (\%) }\end{array}$ \\
\hline \multicolumn{4}{|l|}{ Clinical } \\
\hline acute cutaneous & 435 & 425 & $98 \%$ \\
\hline Chronic cutaneous & 146 & 141 & $97 \%$ \\
\hline Renal & 182 & 118 & $65 \%$ \\
\hline Serositis & 221 & 115 & $52 \%$ \\
\hline Arthritis & 472 & 161 & $34 \%$ \\
\hline Neurological & 205 & 59 & $29 \%$ \\
\hline Oral ulcers & 281 & 46 & $16 \%$ \\
\hline Alopecia & 96 & 1 & $1 \%$ \\
\hline \multicolumn{4}{|l|}{ LABORATORY } \\
\hline Anti-dsDNA Ab & 348 & 310 & $89 \%$ \\
\hline Hemolytic Anemia & 5 & 4 & $80 \%$ \\
\hline Complement & 500 & 368 & $74 \%$ \\
\hline $\begin{array}{l}\text { Leukopenia/ } \\
\text { Lymphopenia }\end{array}$ & 508 & 369 & $73 \%$ \\
\hline $\begin{array}{l}\text { Anti-phospholipid } \\
\text { antibody }\end{array}$ & 131 & 84 & $64 \%$ \\
\hline $\begin{array}{l}\text { Anti-nuclear } \\
\text { antibody }\end{array}$ & 433 & 226 & $52 \%$ \\
\hline Thrombocytopenia & 454 & 98 & $22 \%$ \\
\hline Coombs & 12 & 2 & $17 \%$ \\
\hline Anti-Smith antibody & 109 & 0 & $0 \%$ \\
\hline
\end{tabular}

Conclusions Query of patient EHR data with ICD-9/10 codes and lab tests for specific SLICC classification criteria items requires refinement to improve identification of some criteria. Using the SLICC classification rule for definite SLE, we were able to identify $88 \%$ of those with definite SLE by the ACR criteria, using ICD9/ 10 codes and labs. Text searching of notes (by simple string matching or natural language processing) may improve identification of individual SLICC criteria (e.g. renal biopsy) and may be critical for mining physicians' notes for criteria that are not well documented with diagnosis codes or lab results.

Acknowledgements Funding-NIH/NIAMS R21AR074081.

\section{BD-10 THE DISTRIBUTION OF INSURANCE IN A POPULATION- BASED COHORT OF SLE: GEORGIANS ORGANIZED AGAINST LUPUS COHORT}

S Sam Lim*, Gaobin Bao, Cristina Drenkard. Emory University, Department of Medicine, Division of Rheumatology, Atlanta, Georgia USA

\subsection{6/lupus-2018-Ism.34}

Background Having health insurance coverage is important for people with chronic conditions. Those with systemic lupus erythematosus (SLE) are particularly vulnerable given the disproportionate impact on young minorities and women. This is the first description of health insurance changes over time overall and by sociodemographic groups on a population level. 